\title{
Exploring the link between behaviour and health
}

\author{
Franz Gravenhorst • Venet Osmani • \\ Bert Arnrich · Amir Muaremi
}

Published online: 25 September 2014

(C) Springer-Verlag London 2014

\section{Introduction}

Mobile and wearable sensors are increasingly permeating our lives, and information gathered from them can provide unprecedented insights into diverse aspects of human behaviour. Analysis of human behaviour is of special interest in health care, as there exists dual relationship between behaviour and health.

On one hand, our health is influenced by our behaviour, including physical activity levels, amount of social activity, and work-life balance amongst others, while on the other hand, symptoms of various disorders are manifested as behaviour changes. This is especially prominent for mental disorders [11]. Therefore, human behaviour understanding has significant value for health care, from the point of view of both maintaining good health and helping in the diagnosis of the diseases.

While the link between various aspects of behaviour and health has been explored in clinical settings, use of technology to automatically measure behaviour is still in its infancy. Considering enormous potential of automatic

F. Gravenhorst · A. Muaremi

Wearable Computing Laboratory, ETH Zurich,

Zurich, Switzerland

e-mail: gravenhorst@ife.ee.ethz.ch

A. Muaremi

e-mail: muaremi@ife.ee.ethz.ch

V. Osmani $(\bowtie)$

CREATE-NET, Trento, Italy

e-mail: venet.osmani@create-net.org

B. Arnrich

Bogazici University, Istanbul, Turkey

e-mail: bert.arnrich@boun.edu.tr behaviour understanding in health care, this Theme Issue explores the link between automatic understanding of human behaviour and how it can inform decisions of range of stakeholders in the health ecosystem. Sensing modalities, data processing methods, and behaviour capturing techniques that facilitate this exploration received a particular focus in the contents of this Theme Issue.

As such, authors in [8] present an automated behaviour analysis system, consisting of a sensor network set-up in a home setting. Experiments performed showed how sensor readings can be used to automatically detect anomalous behaviour. This anomalous behaviour can be a sign of health changes in the user, and automatic detection could offer the possibility for intervention if required.

In the same theme of detecting anomalous behaviour, authors in [5] propose an activity recognition system based on the Markov logic network. The performance and use of the method in dementia care is demonstrated by applying it to a dataset recorded in a smart home environment. Results indicate that the hierarchical approach presented has higher accuracy in recognition and a faster response time than existing approaches.

As one of the first step in detecting activities, segmentation of data is typically required. In this regard, the paper in [9] presents an approach that enables segmentation of continuous sensor data in real time. The proposed dynamic segmentation is based on a two-layer strategy-sensor correlation and time correlation manipulation. The methodology was validated utilising two different datasets recorded in smart home settings.

Performance measurement of machine learning methods in order to understand human behaviour was considered in [1]. The authors have evaluated the performance of two machine learning methods on five real-world datasets. They show that the commonly used metrics such as 
accuracy or f-measure are not suitable for datasets in this specific domain. Instead, they propose and demonstrate an alternative evaluation mechanism that considers three different activity categories.

Analysis of diverse behaviour aspects is also of interest in clinical settings, such as hospitals. This is the topic of work in [10], where authors present a solution that enables the capture of multimodal activity in real-world, clinical settings. Their proposed Lab-in-a-Box system includes multiple sensors and is optimised for use in medical office environments. It can collect, synchronise, and segment data describing computer-based activity, speech interaction, visual attention, and body movements. The authors understand their system as a framework to enable more indepth analysis of medical activities.

Outside of clinical setting, high penetration of mobile phones, their sensing capabilities, and feedback modalities they offer make mobile phones promising devices to measure the behaviour of patients in their daily life. The authors in [6] provide an overview of how mobile phones can support the treatment for mental disorders, focusing on the design aspects of human-computer interfaces, technical possibilities for data collection, and post-processing methods. The work in this paper summarises advantages and drawbacks of the most promising mobile phone technologies for detecting mood disorders. Practical aspects such as legal issues and possible business models are also discussed. In the same theme of monitoring patients with mental disorders, authors in [4] describe how machine learning techniques can be used to determine which behavioural data have the highest impact on patients' health. The rankings acquired from their algorithms correspond to the patients' rankings, identifying physical activity and sleep as the highest impact parameters.

While there has been work done in understanding mobile phone usage for the general population, the specific ways in which patients use their phones are less understood. The paper "Cell Phone Ownership and Use amongst Mental Health Outpatients in the US" [2] presents the results of a survey of 325 patients currently receiving treatment at outpatient clinics for mental illnesses. Patients were interviewed regarding their cell phone use and ownership. The results were compared to figures obtained from a nationally representative sample and similarities, and differences were explored. Based on their results, the authors provide lessons on mental disorder treatment using mobile phones.

On the topic of behaviour of elderly, the paper "Behavioural Data Gathering for Assessing Functional Status and Health in Older Adults using Mobile Phones" [3] presents InCense, a toolkit for mobile phones which enables behaviour data collection. The authors present components and features of the toolkit and present results of a data collection study they performed with 15 elderly people. The results show how the data can be used to support functional assessment of the elderly and potentially substitute less objective and unreliable self-reports by patients.

The final paper "e-ESAS: Evolution of a Participatory Design based Solution for Breast Cancer (BC) Patients in Rural Bangladesh" describes the potential and challenges of pervasive health care in developing countries [7]. The authors performed a study with 39 breast cancer patients in rural Bangladesh, where the patients were equipped with low-cost mobile phones running symptom monitoring system. The authors describe the lessons learned concerning the design of this application.

Evidently, this Theme Issue considered understanding of human behaviour from diverse aspects, using diverse methodologies and the impact of behaviour understanding on health. This is a topic that will gain increasing significance as the benefits of behaviour monitoring are better understood, while monitoring technologies increase in capability.

This Theme Issue received over 32 submissions, and while we could accept only 10, we would like to thank authors and reviewers for their valuable contribution. We hope that the papers in this theme issue will become useful references to researchers and practitioners in the field.

\section{References}

1. Alemdar H, Tunca C, Ersoy C (2014) Daily life behaviour monitoring for health assessment using machine learning: bridging the gap between domains. Pers Ubiquit Comput. doi:10. 1007/s00779-014-0823-y

2. Campbell B, Caine K, Connelly K, Doub T, Bragg A (2014) Cell phone ownership and use among mental health outpatients in the US. Pers Ubiquit Comput. doi:10.1007/s00779-014-0822-z

3. Castro LA, Favela J, Quintana E, Perez M (2014) Behavioral data gathering for assessing functional status and health in older adults using mobile phones. Pers Ubiquit Comput. doi:10.1007/s00779014-0825-9

4. Doryab A, Frost M, Faurholt-Jepsen M, Kessing LV, Bardram JE (2014) Impact factor analysis: combining prediction with parameter ranking to reveal the impact of behavior on health outcome. Pers Ubiquit Comput. doi:10.1007/s00779014-0826-8

5. Gayathri KS, Elias S, Ravindran B (2014) Hierarchical activity recognition for dementia care using markov logic network. Pers Ubiquit Comput. doi:10.1007/s00779-014-0827-7

6. Gravenhorst F, Muaremi A, Bardram J, Grünerbl A, Mayora O, Wurzer G, Frost M, Osmani V, Arnrich B, Lukowicz P, Tröster $\mathrm{G}$ (2014) Mobile phones as medical devices in mental disorder treatment: an overview. Pers Ubiquit Comput. doi:10.1007/ s00779-014-0829-5

7. Haque MM, Kawsar F, Adibuzzaman M, Uddin MM, Ahamed SI, Love R, Hasan R, Dowla R, Ferdousy T, Salim R (2014) e-ESAS: 
Evolution of a participatory design-based solution for breast cancer (BC) patients in rural Bangladesh. Pers Ubiquit Comput. doi:10.1007/s00779-014-0828-6

8. Ordóñez FJ, de Toledo P, Sanchis A (2014) Sensor-based bayesian detection of anomalous living patterns in a home setting. Pers Ubiquit Comput. doi:10.1007/s00779-014-0820-1

9. Wan J, O'Grady MJ, O'Hare GMP (2014) Dynamic sensor event segmentation for real-time activity recognition in a smart home context. Pers Ubiquit Comput. doi:10.1007/s00779-014-0824-x
10. Weibel N, Rick S, Emmenegger C, Ashfaq S, Calvitti A, Agha Z (2014) Lab-in-a-box: semi-automatic tracking of activity in the medical office. Pers Ubiquit Comput. doi:10.1007/s00779-0140821-0

11. Gruenerbl A, Muaremi A, Osmani V, Bahle G, Oehler S, Troester G, Mayora O, Haring C, Lukowicz P (2014) Smart-phone based recognition of states and state changes in bipolar disorder patients. IEEE J Biomed Health Inform. doi:10.1109/JBHI.2014. 2343154 